\title{
Ultrasound Shear Wave Elastography Helps Discriminate Low-grade From High-grade Bowel Wall Fibrosis in Ex Vivo Human Intestinal Specimens
}

\author{
Jonathan R. Dillman, MD, Ryan W. Stidham, MD, Peter D. R. Higgins, MD, PhD, MSc, David S. Moons, MD, \\ PhD, Laura A. Johnson, BS, Nahid R. Keshavarzi, MSc, Jonathan M. Rubin, MD, PhD
}

Received January 30, 2014, from the Departments of Radiology (J.R.D., J.M.R.), Internal Medicine, Division of Gastroenterology (R.W.S., P.D.R.H., L.A.J.), and Pathology (D.S.M.), University of Michigan Health System, Ann Arbor, Michigan USA; and Michigan Institute for Clinical and Health Research, University of Michigan, Ann Arbor, Michigan USA (N.R.K.). Revision requested March 13, 2014. Revised manuscript accepted for publication March 20, 2014.

This study was supported by Siemens Medical Solutions USA, Inc, in the form of an investigator-initiated grant as well as by grant 2UL1TR000433 from the National Center for Advancing Translational Sciences (NCATS). The content is solely the responsibility of the authors and does not necessarily represent the official views of the NCATS or the National Institutes of Health.

Address correspondence to Jonathan $R$. Dillman, $M D$, Department of Radiology, Section of Pediatric Radiology, University of Michigan Health System, C. S. Mott Children's Hospital, 1540 E Hospital Dr, Ann Arbor, MI 48109-4252 USA.

E-mail: jonadill@med.umich.edu

\section{Abbreviations}

CI, confidence interval; ROC, receiver operating characteristic; VT-IQ Virtual TouchIQ; VTQ, Virtual Touch quantification

doi: $10.7863 /$ ultra.33.12.2115
Objectives - To determine whether bowel wall fibrosis can be detected in freshly resected human intestinal specimens based on ultrasound-derived shear wave speed.

Methods - Seventeen intact (>3-cm) bowel segments (15 small and 2 large intestine) from 12 patients with known or suspected inflammatory bowel disease were procured immediately after surgical resection. Ultrasound shear wave elastography of the bowel wall was performed by two methods (Virtual Touch Quantification [VTQ] and Virtual Touch-IQ [VT-IQ]; Siemens Medical Solutions USA, Inc, Mountain View, $\mathrm{CA}$ ). Eighteen short-axis shear wave speed measurements were acquired from each specimen: 3 from the 9-, 12-, and 3-o'clock locations for each method. Imaging was performed in two areas for specimens greater than $10 \mathrm{~cm}$ in length (separated by $\geq 5$ $\mathrm{cm}$ ). A gastrointestinal pathologist scored correlative histologic slides for inflammation and fibrosis. Differences in mean shear wave speed between bowel segments with low and high inflammation/fibrosis scores were assessed by a Student $t$ test. Receiver operating characteristic curve analysis was performed.

Results-High-fibrosis score $(\mathrm{n}=11)$ bowel segments had a significantly greater mean shear wave speed than low-fibrosis score $(n=6)$ bowel segments (mean \pm SD: VTQ $1.59 \pm 0.37$ versus $1.18 \pm 0.08 \mathrm{~m} / \mathrm{s} ; P=.004$; VT-IQ $1.87 \pm 0.44$ versus $1.50 \pm 0.26 \mathrm{~m} / \mathrm{s}$; $P=.049)$. There was no significant difference in mean shear wave speed between highand low-inflammation score bowel segments $(P>.05$ for both VTQ and VT-IQ). Receiver operating characteristic curves showed areas under the curve of 0.91 (95\% confidence interval, 0.67-0.99) for VTQ and 0.77 (95\% confidence interval, 0.51-0.94) for VT-IQ in distinguishing low- from high-fibrosis score bowel segments.

Conclusions - Ex vivo bowel wall shear wave speed measurements increase when transmural intestinal fibrosis is present.

Key Words - Crohn disease; fibrosis; gastrointestinal ultrasound; intestine; shear wave elastography

\footnotetext{
C rohn disease is a relapsing and remitting inflammatory condition affecting the human gastrointestinal tract that affects approximately 600,000 persons in North America and is associated with substantial morbidity and health care costs. ${ }^{1-3}$ Fibrosis, a final common pathway of chronic injury in many organs, occurs unpredictably in both untreated and treated patients with Crohn disease and may be complicated by focal intestinal narrowings (strictures) and perforations (fistulas). ${ }^{4-6}$ Approximately $64 \%$ of
} 
adult patients with Crohn disease have intestinal strictures, fistulas (which are commonly associated with strictures), or both requiring surgery after 30 years. ${ }^{7}$

Strictures of the bowel lumen in Crohn disease that cause mural thickening and luminal narrowing may be due to inflammation or fibrosis. A recent study by Adler et al ${ }^{8}$ documented that most Crohn disease strictures contain both inflammation and fibrosis, and, somewhat unexpectedly, highly fibrotic strictures also frequently contain large amounts of superimposed active inflammation. The same study also demonstrated that computed tomographic enterography cannot reliably establish the presence of bowel wall fibrosis in Crohn disease. ${ }^{8}$ Detecting the presence of bowel wall fibrosis is important as intestinal segments that are narrowed due to inflammation generally respond to medical therapy, whereas narrowed fibrotic intestinal segments are generally unresponsive to currently available medications, thus frequently requiring surgery. 9,10

Computed tomography and magnetic resonance enterography are radiologic tests commonly used to evaluate patients with Crohn disease that accurately identify affected bowel segments as well as provide information about inflammatory activity and complications. Unfortunately, these diagnostic modalities cannot reliably determine whether areas of focal intestinal narrowing and obstruction in patients with Crohn disease are due to the presence of mural fibrosis, especially when inflammation and fibrosis are coexistent. The development and optimization of imaging technologies that can detect intestinal fibrosis even when inflammation is present as well as quantify the amount of fibrosis would almost certainly cause fundamental paradigm shifts in the radiologic assessment and clinical (both medical and surgical) management of Crohn disease in children and adults. Knowledge of the amount of bowel wall fibrosis would allow clinicians to identify patients best served by immediate surgical therapy, thus possibly avoiding the ineffectual use of often costly, sometimes harmful immunosuppressant and anti-inflammatory medical therapies. Such personalized management of heath care has the potential to improve outcomes in patients with Crohn disease.

We postulate that shear wave elastographic (acoustic radiation force impulse-derived shear wave speed) measurements can provide noninvasive insight into the histologic composition of strictured intestinal segments in Crohn disease based on biomechanical elastic properties, even when both inflammation and fibrosis are present. Fibrosis is harder (stiffer) than inflammation (a large component of which is edema, or water); thus, strictures containing considerable fibrosis should be associated with higher intramural shear wave speed measurements than purely inflammatory strictures. On the basis of the bowel wall shear wave speed, it may be possible to establish the presence of fibrosis at the time of initial Crohn disease diagnosis or stricture presentation. Increasing bowel wall shear wave speed over time may indicate developing or progressive fibrosis. A change in shear wave speed over time also may serve as a quantitative radiologic biomarker for medical therapy response (or failure). A recent study by Dillman et $\mathrm{al}^{11}$ showed that bowel wall shear wave speed measurements can be used to distinguish fibrotic from nonfibrotic colon in a Crohn disease rat model both without and with increasing amounts of applied strain (transducer preload).

We must now translate intestinal shear wave elastography from the animal model into humans. The purpose of this exploratory investigation was to determine whether bowel wall fibrosis can be detected in surgically resected human intestinal specimens based on ex vivo intramural shear wave speed measurements. We hypothesized that resected bowel specimens containing extensive (transmural) fibrosis are physically harder and will have higher shear wave speed measurements than those bowel specimens that are inflamed and that contain no or minimal fibrosis. In a linear regimen and assuming material isotropy, material (tissue) hardness is proportional to the square of shear wave speed. ${ }^{12}$

\section{Materials and Methods}

Institutional Review Board approval was obtained for this investigation. Resected bowel specimens were obtained with the assistance of the University of Michigan Tissue Procurement Service.

Pediatric and adult small- and large-bowel specimens from individuals with suspected or known inflammatory bowel disease were procured fresh immediately after surgical resection between June 2012 and May 2013 and taken to a Department of Pathology laboratory for sonography. Care was taken to ensure that the resected bowel specimens were not placed in formalin or any other chemical tissue preservative/fixative. Resected bowel specimens were placed on a standoff pad (Aquaflex ultrasound gel pad; Parker Laboratories, Inc, Fairfield, NJ) that rested on a laboratory bench and bathed in copious amounts of ultrasound gel (Aquasonic 100; Parker Laboratories, Inc).

After grayscale sonography of resected bowel specimens in the long and short axes, shear wave elastography was performed in the area of greatest wall thickening by two different methods (Virtual Touch quantification [VTQ] and Virtual Touch-IQ [VT-IQ]; Acuson S3000 ultrasound system; Siemens Medical Solutions USA, Inc, 
Mountain View, CA; Figure 1). Both VTQ and VT-IQ are recently developed forms of quantitative shear wave elastographic imaging. Virtual Touch quantification uses an acoustic radiation force impulse "push pulse" $(4.00 \mathrm{MHz})$ and provides a measurement of shear wave speed within a single small region of interest. Tracking beams are applied adjacent to the acoustic push pulse, and the time between generation of the shear wave and the passing shear wave peak at an adjacent location is used to calculate the shear wave speed. Virtual Touch-IQ uses multiple push pulses (4.44 or $5.71 \mathrm{MHz}$, depending on the depth of imaging within tissue) across the transducer face and at multiple depths and provides a color image (elastogram) of tissue hardness based on the shear wave speed. Multiple regions of interest can be placed on the color image, allowing for multiple shear wave speed measurements.
Using a 9L4 linear matrix transducer and minimal operator transducer pressure/preload (essentially $0 \%$ applied strain, as transducer pressure applied to the specimens was only due to the force of gravity acting on the transducer), shear wave elastography of the bowel wall was performed by a single operator (J.R.D.) with the transducer oriented perpendicular to the long axis of the intestinal lumen. Bowel wall shear wave speed measurements initially were acquired from each resected intestinal segment (3 measurements from the 9-, 12-, and 3-o'clock locations, respectively) using VTQ technology and 9 separate image acquisitions (Figure 1C). Next, 9 similarly located shear wave speed measurements were acquired from the bowel wall using VT-IQ and 3 separate image acquisitions ( 3 shear wave speed measurements per image; Figure 1D). For intestinal specimens with contiguous

Figure 1. Images from a 23-year-old man with stricturing, fistulizing Crohn disease. A and B, Long-and short-axis grayscale images of resected terminal ileum showing bowel wall thickening (arrows) and adjacent mesenteric fibrofatty proliferation, or "creeping fat" (asterisks). A blind-ending sinus tract is shown on the short-axis image (arrowheads). SP indicates standoff pad. C and D, Short-axis VTQ and VT-IQ shear wave elastographic images showing placement of regions of interest in the bowel wall at approximately the 3-o'clock position. The VT-IQ image provides a color elastogram of tissue hardness based on shear wave speed. A small amount of echogenic, shadowing air is present in the bowel lumen (arrows).

A

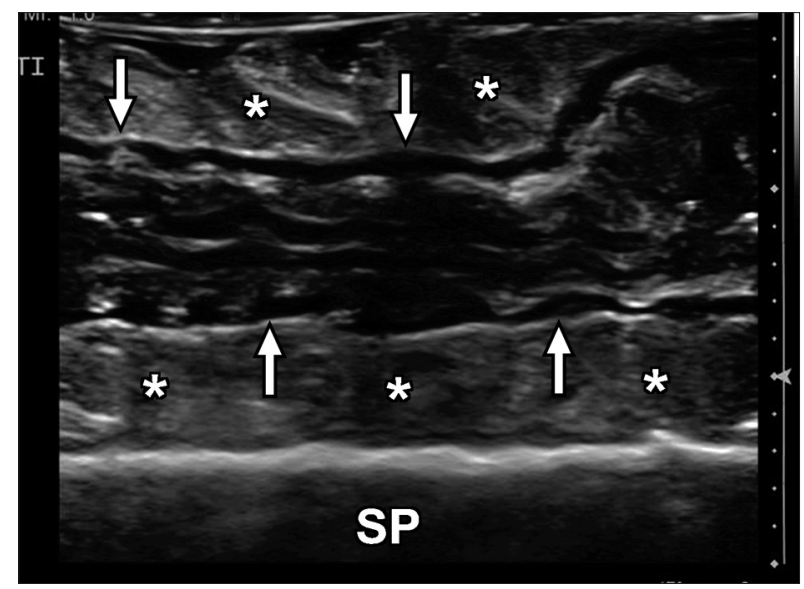

C

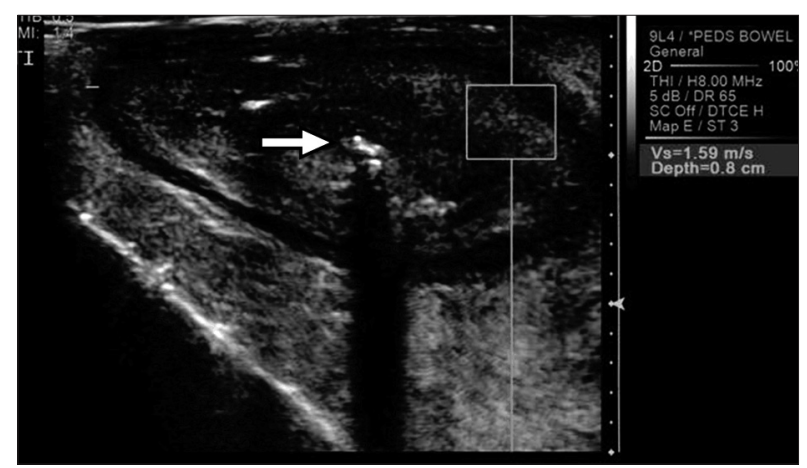

B

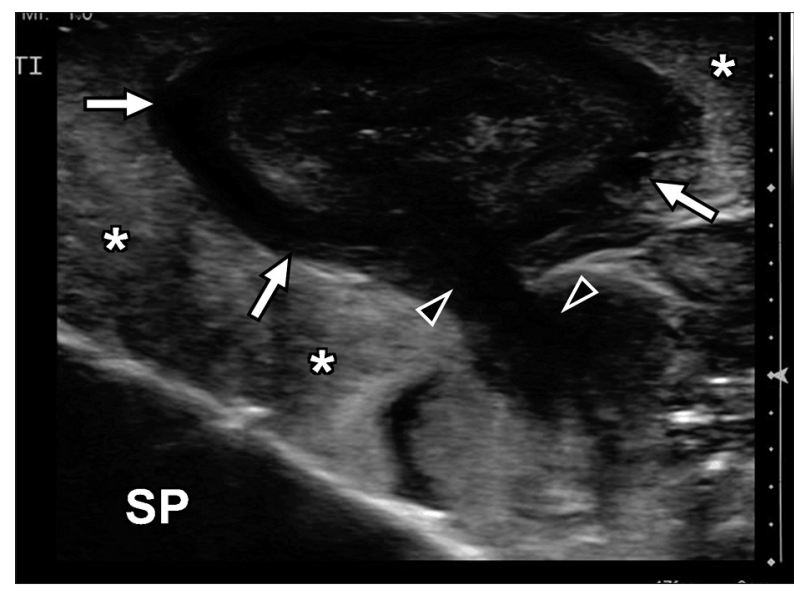

D

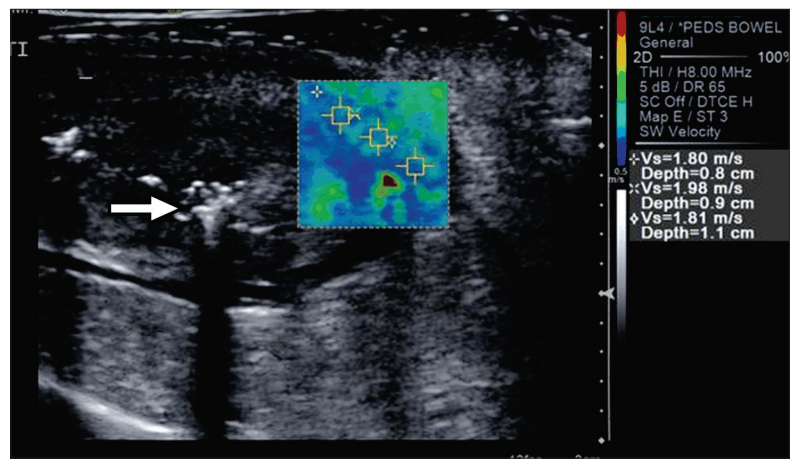


abnormalities measuring greater than $10 \mathrm{~cm}$ in length, shear wave elastography was performed in two different areas, separated by a minimum of $5 \mathrm{~cm}$.

Full-thickness bowel wall tissue from the sonographic areas was obtained for histopathologic evaluation. After standard tissue processing, a gastrointestinal pathology specialist evaluated histologic specimens (hematoxylin-eosin- as well as Masson trichrome-stained slides) for both inflammation and fibrosis using Likert-like scales based on previously described scoring systems, while blinded to the sonographic findings. ${ }^{13,14}$ The Likert-like scoring systems used to assess bowel wall inflammation and fibrosis are presented in Tables 1 and 2 .

Statistical analysis was performed by the study statistician. An unpaired 2-tailed Student $t$ test (assuming unequal variances) was used to assess differences in mean shear wave speed between bowel segments with low and high inflammation scores ( $0-2$ versus 3 and 4$)$ and low and high fibrosis scores ( $0-2$ versus 3$)$. The relationships between mean shear wave speed and both inflammation and fibrosis scores were assessed by Spearman rank correlation $(\rho)$. Kendall rank correlation $(\tau)$ and Pearson correlation $(r)$ were used to explore the relationships between bowel wall inflammation and fibrosis (based on Likert-like scoring) and between shear wave speed measurements obtained by VTQ and VT-IQ from the same resected bowel segment, respectively. Receiver operating characteristic (ROC) curve analysis was performed to assess the diagnostic performance of shear wave elastography for detecting extensive bowel wall fibrosis (fibrosis score 3). When shear wave speed measurements were acquired from two separate areas within the same resection specimen, measurements were considered independent, as inflammatory bowel disease is a heterogeneous process, and imaged bowel segments were separated by greater than $5 \mathrm{~cm}$. $P<.05$ was considered statistically significant for hypothesis testing. Statistical analyses were performed with Stata 12.1 software (StataCorp LP, College Station, TX).

\section{Results}

Seventeen intact $(>3-\mathrm{cm})$ bowel segments $(15$ small intestine and 2 large intestine) from 12 patients with known or suspected inflammatory bowel disease were procured fresh immediately after surgical resection (Table 3 ). Six patients were male, and 6 were female. The mean patient age was 24.9 years (range, $10-53$ years).

\section{Mean Shear Wave Speed}

High-fibrosis score $(\mathrm{n}=11)$ bowel segments had a significantly greater mean shear wave speed than low-fibrosis score $(\mathrm{n}=6)$ bowel segments (mean \pm SD: VTQ $1.59 \pm$ 0.37 versus $1.18 \pm 0.08 \mathrm{~m} / \mathrm{s} ; P=.004$; VT-IQ $1.87 \pm$ 0.44 versus $1.50 \pm 0.26 \mathrm{~m} / \mathrm{s} ; P=.049$; Figures $1-3$ ). The area under the ROC curve for VTQ in differentiating high- versus low-fibrosis score bowel segments was 0.91 (95\% confidence interval $[\mathrm{CI}], 0.67-0.99 ; P<.0001$ ), and the area under the ROC curve for VT-IQ was 0.77 (95\% CI, $0.51-0.94 ; P=.025$; Figure 4 ). There was no significant difference in mean shear wave speed between high-score and low-inflammation score bowel segments (VTQ $1.60 \pm 0.50$ versus $1.33 \pm 0.19 \mathrm{~m} / \mathrm{s} ; P=.22$; VT-IQ $1.86 \pm 0.54$ versus $1.66 \pm 0.32 \mathrm{~m} / \mathrm{s} ; P=.4$ ).

Table 1. Histologic Scoring System for Active Inflammation in Resected Human Intestinal Specimens

\begin{tabular}{ll}
\hline Score & \multicolumn{1}{c}{ Degree of Inflammation } \\
\hline 0 (none) & No inflammation \\
1 & Low level of inflammation with scattered infiltrating mononuclear cells \\
2 & Moderate inflammation with multiple foci \\
3 & High level of inflammation with increased vascular density and marked wall thickening \\
4 (severe) & Maximal severity of inflammation with transmural leukocyte infiltration and loss of goblet cells \\
\hline
\end{tabular}

Table 2. Histologic Scoring System for Fibrosis in Resected Human Intestinal Specimens

\begin{tabular}{ll} 
Score & \multicolumn{1}{c}{ Degree of Fibrosis } \\
\hline 0 (none) & No architectural distortion, no abnormal Masson trichrome staining \\
1 & No architectural distortion, mild abnormal Masson trichrome staining in mucosa/submucosa \\
2 & $\begin{array}{c}\text { Substantial abnormal mucosal/submucosal Masson trichrome staining with modest distortion of architecture } \\
\text { but without obscuration of the mucosal/submucosal border } \\
\text { Transmural fibrosis with abnormal Masson trichrome staining in all histologic layers, transmural architectural } \\
\text { distortion }\end{array}$
\end{tabular}




\section{Relationship Between Mean Shear Wave Speed and Fibrosis and Inflammation Scores}

A moderate correlation was observed between an increasing bowel wall shear wave speed and an increasing fibrosis score (VTQ $\rho=0.60$; VT-IQ $\rho=0.47$; Figure 5). The relationship was significant for the VTQ method $(P=.01)$ of shear wave speed measurement and marginally significant for the VT-IQ method $(P=.057)$. The relationship between bowel wall shear wave speed and inflammation was less correlative and nonsignificant (VTQ, $\rho=0.38 ; P=.13$; VT-IQ $\rho=0.24 ; P=.36$ ).

\section{Relationship Between Bowel Wall Inflammation and Fibrosis}

Histologic scoring demonstrated that bowel wall inflammation and fibrosis commonly coexist in resected bowel specimens (Table 3). A moderately linear, significant relationship was observed $(\tau=0.45 ; P=.01)$.

\section{Relationship Between VTQ and VT-IQ Shear Wave Speed Measurements}

On average, mean bowel wall shear wave speed measurements were higher when using VT-IQ compared to VTQ $(1.74 \pm 0.42$ versus $1.44 \pm 0.36 \mathrm{~m} / \mathrm{s})$, with a statistically

Table 3. Patient Demographic Data and Inflammation/Fibrosis Scores

\begin{tabular}{|c|c|c|c|c|c|c|}
\hline Patient & Age, $y$ & Sex & CD vs UC & $\begin{array}{l}\text { Imaged } \\
\text { Bowel }\end{array}$ & $\begin{array}{c}\text { Fibrosis } \\
\text { Score }^{a}\end{array}$ & $\begin{array}{l}\text { Inflammation } \\
\text { Score }\end{array}$ \\
\hline 1 & 23 & Male & $C D$ & lleum, AC & 3,0 & 3,0 \\
\hline 2 & 17 & Female & UC & Ileum & 0 & 1 \\
\hline 3 & 10 & Female & UC & $A C$ & 0 & 0 \\
\hline 4 & 52 & Female & $C D$ & Ileum (×2) & 3,3 & 2,1 \\
\hline 5 & 30 & Female & $C D$ & Ileum $(\times 2)$ & 3,3 & 1,2 \\
\hline 6 & 10 & Female & UC & Ileum & 1 & 0 \\
\hline 7 & 27 & Male & $C D$ & Ileum (×2) & 3,3 & 3,3 \\
\hline 8 & 30 & Male & $C D$ & Ileum & 3 & 4 \\
\hline 9 & 15 & Male & $C D$ & Ileum & 3 & 4 \\
\hline 10 & 53 & Male & b & Ileum & 2 & 1 \\
\hline 11 & 19 & Female & $C D$ & Ileum & 3 & 4 \\
\hline 12 & 13 & Male & $C D$ & Ileum $(\times 2)$ & 3,2 & 2,4 \\
\hline
\end{tabular}

AC indicates ascending colon; $C D$, Crohn disease; and UC, ulcerative colitis.

aFor intestinal specimens measuring greater than $10 \mathrm{~cm}$ in length, shear wave elastography and histologic scoring were performed in two different areas, separated by a minimum of $5 \mathrm{~cm}$.

'The patient had suspected terminal ileitis due to Crohn disease, although histopathologic evaluation confirmed ileitis (bowel wall inflammation and fibrosis) due to an adjacent carcinoid tumor.

Figure 2. Images from a 20-year-old man with stricturing Crohn disease. A and B, Short-axis VTQ and VT-IQ shear wave elastographic images showing placement of regions of interest in the bowel wall at approximately the 3-o'clock position. There is severe bowel wall thickening and heterogeneity on the underlying grayscale images. The small bowel mesentery is markedly thickened.

A

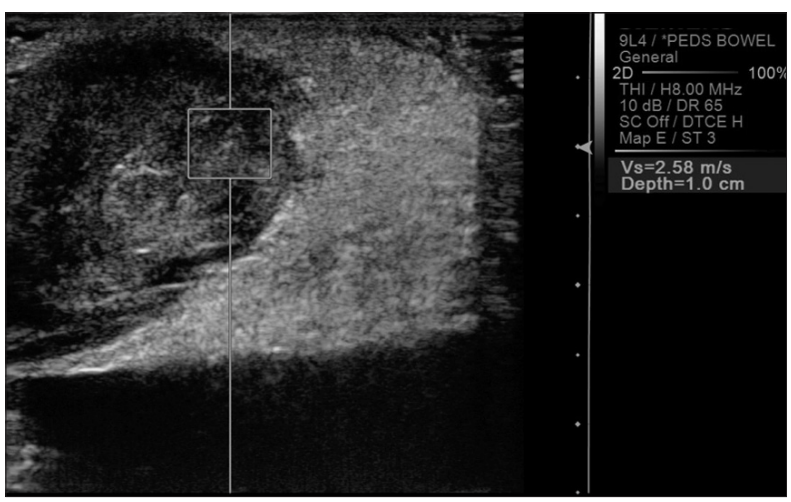

B

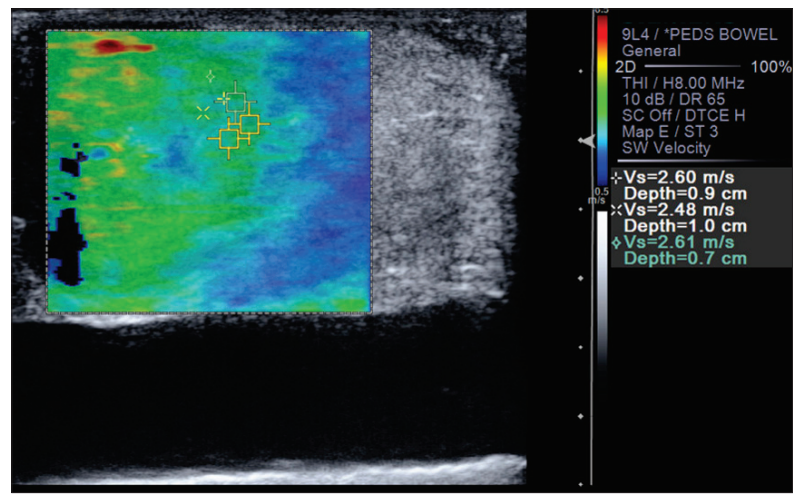


significant difference $(P=.04$, paired Student $t$ test $)$. There was a significant moderate correlation between bowel wall shear wave speed measurements obtained by VTQ and VT-IQ $(r=0.57 ; P=.02)$.

Figure 3. Box plot comparing mean shear wave speed (SWS) measurements for low-versus high-fibrosis score bowel segments obtained by the VTQ shear wave elastographic method. Whiskers represent minimum and maximum shear wave speed measurements.

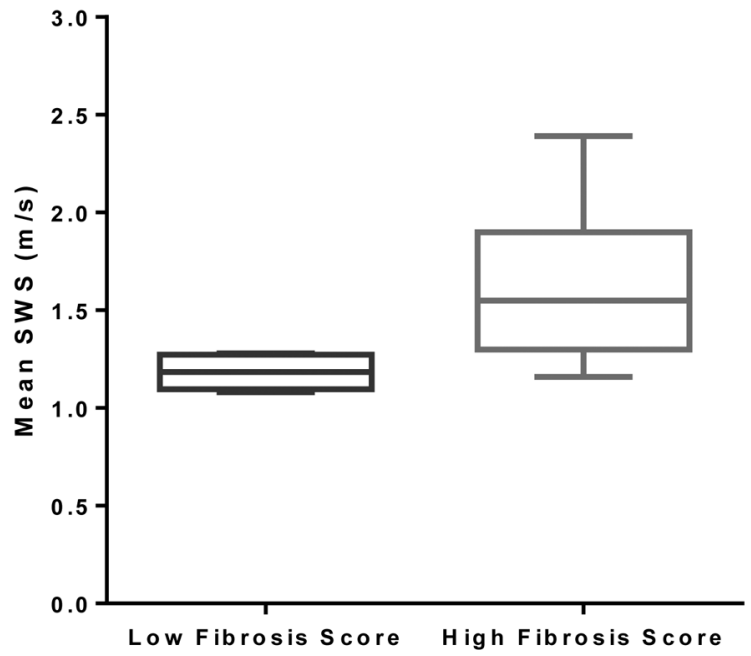

\section{Discussion}

A noninvasive diagnostic tool that could reliably establish the histologic composition of narrowed, obstructed bowel segments in vivo could impact both the medical and surgical management of Crohn disease. If luminal narrowing could be shown to be due to primarily active inflammation, then the treating physician would likely pursue medical management in the form of anti-inflammatory and immunosuppressive medications. On the other hand, if luminal narrowing could be shown to be due to primarily fibrosis, then the treating physician would likely pursue surgical management or endoscopic intervention. Unfortunately, there is currently no such diagnostic tool available.

A small number of recent studies have investigated the use of ultrasound elasticity imaging for discriminating bowel wall inflammation from fibrosis. ${ }^{11,15-17}$ Both Kim et $\mathrm{al}^{15}$ and $\mathrm{Xu}$ et a ${ }^{16}$ showed in Crohn disease animal models that strain developed under quasistatic deformation can be used to distinguish inflamed from fibrotic intestine. A recent study by Stidham et al ${ }^{17}$ also used quasistatic strain imaging to successfully differentiate normal from fibrotic bowel in vivo in 7 humans with Crohn disease.

Shear wave elastography is an innovative form of ultrasound elasticity imaging that is becoming increasingly clinically available throughout the world, including the United States. This noninvasive transcutaneous technique allows for quantitative estimation of tissue hardness based on the speed of shear (or transverse) wave propagation in tissues

Figure 4. Receiver operating characteristic curves for discriminating low-from high-fibrosis score bowel segments. A, Virtual Touch quantification shear wave elastographic method (area under the curve, 0.91; 95\% Cl, 0.67-0.99; $P<.0001$ ). B, Virtual Touch-IQ shear wave elastographic method (area under the curve, $0.77 ; 95 \% \mathrm{Cl}, 0.51-0.94 ; P=.025$ ).

A

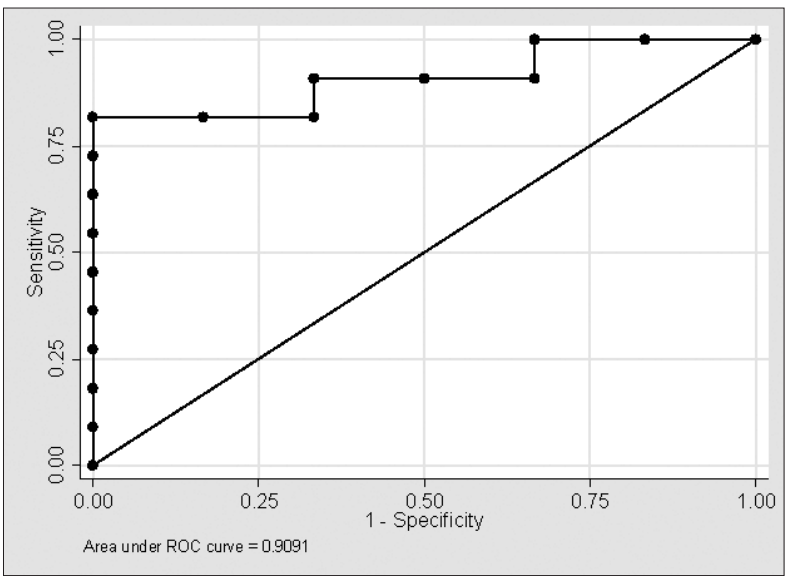

B

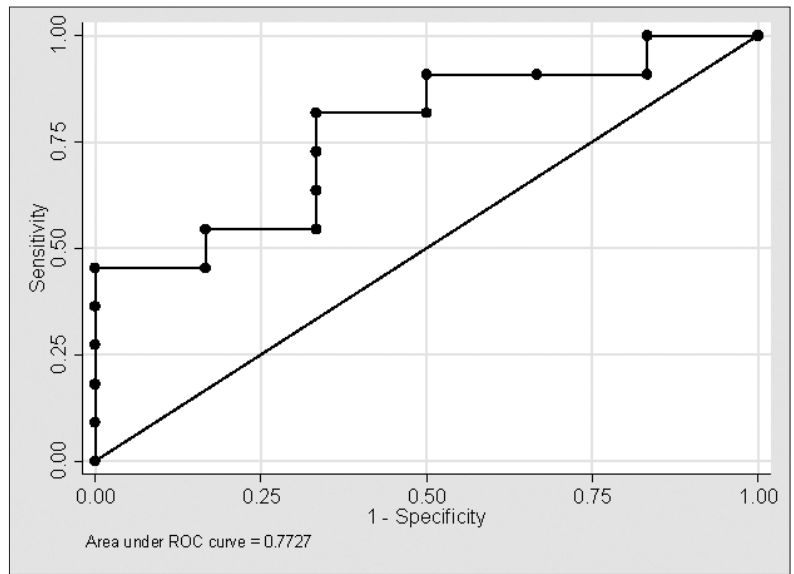


of interest after an acoustic radiation force impulse, or push pulse. The shear wave speed is related to the shear modulus and has a wide dynamic range in human tissues. ${ }^{18} \mathrm{~A}$ recent study by Dillman et $\mathrm{al}^{11}$ in a Crohn disease rodent model demonstrated that shear wave elastography holds promise for detecting bowel wall fibrosis in humans with Crohn disease. In their animal model, bowel segments without and with fibrosis could be discriminated on the basis of intramural shear wave speed both without and with increasing increments of applied strain to the rat abdomen (increasing amounts of transducer preload). ${ }^{11}$

In this study, resected human bowel segments with extensive transmural fibrosis (fibrosis score 3 ) had significantly higher mean shear wave speed measurements than those with low fibrosis scores in both VTQ and VT-IQ shear wave elastographic methods. Although a close look at our data shows that there is incomplete separation of high- and low-fibrosis score bowel segments based on the mean shear wave speed, this overlap is minimal. Receiver operating characteristic curve analyses for discriminating bowel segments with high and low fibrosis scores show promising diagnostic performance, with a VTQ area under the ROC curve of greater than 0.9.

The relationship between the bowel wall shear wave speed and fibrosis score was shown to be moderately correlative. Based on scatterplot inspection, the relationship may actually be nonlinear (Figure 5). The mean bowel wall shear wave speed seems to be relatively stable for specimens with fibrosis scores of 0 to 2 , whereas the shear wave speed seems to increase when bowel wall fibrosis becomes extensive and transmural (fibrosis score 3 ). This finding suggests that shear wave elastography may best detect advanced fibrotic changes but may be less capable of differentiating nontransmural mild/moderate fibrosis from early, predominantly inflammatory disease.

Figure 5. Scatterplot showing the relationship between the mean bowel wall shear wave speed (SWS) and histologic fibrosis score by the VTQ shear wave elastographic method ( $\rho=0.60 ; P=.01$ ).

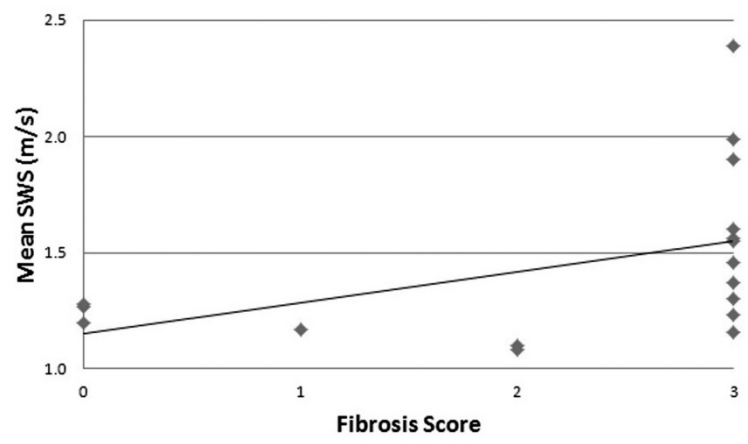

We have confirmed the findings of two previous studies ${ }^{8,19}$ that demonstrated that active and chronic inflammation as well as fibrosis all frequently coexist in bowel segments affected by inflammatory bowel disease. Based on our study, the relationship between inflammation and fibrosis is significant and moderately correlative. Some degree of inflammation was present in all highfibrosis score bowel segments. Therefore, a diagnostic test for detecting bowel wall fibrosis in Crohn disease must be able to perform when inflammation is also present. Based on our results, shear wave elastography seems to be capable of detecting fibrosis even when inflammation is present, unlike conventional sonographic, computed tomographic, and magnetic resonance imaging techniques.

Furthermore, we hypothesize that assessment of the relationship between shear wave speed and increasing applied strain (increasing transducer preload) may allow even better detection of bowel wall fibrosis. Based on the nonlinear elastic properties of tissue fibrosis and inflammation (edema), it is possible that highly fibrotic bowel segments will show a more rapid increase in shear wave speed with increasing applied strain than nonfibrotic, inflamed bowel. This phenomenon, which is likely due to differences in bowel wall collagen and water content, has been demonstrated in a Crohn disease animal model, ${ }^{11}$ and further investigations in human patients are needed. A recent study by Rotemberg et $\mathrm{al}^{20}$ demonstrated that the liver behaves in a nonlinear manner with internal pressurization, with substantial increases in shear wave speed measurements from baseline. Sarvazyan et a ${ }^{18}$ suggested that tissue nonlinearity as assessed by shear wave imaging may be another useful acoustic biomarker that could yield potentially important diagnostic information in human tissues.

Although correlative, mean bowel wall shear wave speed measurements were systematically slightly higher when using VT-IQ compared to VTQ. This difference, which was statistically significant, likely relates to technical differences between the VTQ and VT-IQ methods: for example, the use of a single 4-MHz push pulse versus multiple push pulses at higher frequencies. This observation should be kept in mind going forward, as we use these technologies to characterize the hardness of the bowel as well as other organs and tissues.

As the wall of normal small bowel is "paper thin," its hardness cannot be accurately measured by currently available shear wave imaging methods. Therefore, we were unable to assess normal intestine as part of our investigation. This factor was not a significant limitation, however, as normal bowel can be readily evaluated by multiple con- 
ventional imaging modalities. As we are specifically interested in whether abnormally thickened, obstructed intestinal segments contain fibrosis that is recalcitrant to currently available therapeutics, the fact that the shear wave measurements cannot be obtained from normal bowel is probably inconsequential.

Our study had limitations. First, it was small (17 bowel segments were assessed), and imaging was performed on ex vivo nonperfused intestinal specimens. Even so, this exploratory investigation found compelling, significant results that should be further evaluated in vivo. Second, shear wave elastography, as with other forms of elasticity imaging, is subject to boundary effects and geometric dispersion. As the bowel is a tubular structure composed of different layers with an overall thickness of approximately 3 to greater than $10 \mathrm{~mm}$ in humans with Crohn disease, it is possible that such effects could be present and alter the accuracy of the shear wave speed measurements acquired. Specifically, this situation could result in the generation of guided waves that are a function of both tissue geometry and histologic composition. ${ }^{18}$ However, we presume that these boundary effects, which occur when the wavelength of the shear wave is larger than the imaged object, were consistent across our measurements, and, although they may have affected the absolute shear wave speed measured, the trends observed in our study remain unchanged.

In addition, inflammatory bowel disease affects the bowel wall in a heterogeneous manner both longitudinally and circumferentially. Although bowel wall tissue was obtained for histologic assessment from the site of imaging, it is impossible to have an absolutely perfect one-toone imaging-to-histologic correlation. Finally, blinded histologic Likert-like scoring for bowel wall inflammation and fibrosis was a somewhat subjective process based on the expert opinion of a gastrointestinal pathology specialist.

In conclusion, ex vivo bowel wall shear wave speed measurements increase when intestinal fibrosis is extensive and transmural. Although there is some shear wave speed overlap between low- and high-fibrosis score bowel segments, this overlap is minimal (particularly when using the VTQ shear wave elastographic method). Characterization of intestinal strictures as extensively fibrotic using shear wave elastography has the potential to directly impact medical and surgical decision making in humans with Crohn disease. As our study was exploratory and was performed on ex vivo surgically resected intestinal specimens, further investigations are needed to assess shear wave elastography of human Crohn disease strictures in vivo and to establish whether intestinal fibrosis can be reliably detected on the basis of tissue elastic properties.

\section{References}

1. Kappelman MD, Rifas-Shiman SL, Porter CQ, et al. Direct health care costs of Crohn's disease and ulcerative colitis in US children and adults. Gastroenterology 2008; 135:1907-1913.

2. Loftus EV Jr, Schoenfeld P, Sandborn WJ. The epidemiology and natural history of Crohn's disease in population-based patient cohorts from North America: a systematic review. Aliment Pharmacol Ther 2002; 16:51-60.

3. Bodger K. Cost of illness of Crohn's disease. Pharmacoeconomics 2002; 20:639-652.

4. Loftus CG, Loftus EV Jr, Harmsen WS, et al. Update on the incidence and prevalence of Crohn's disease and ulcerative colitis in Olmsted County, Minnesota, 1940-2000. Inflamm Bowel Dis 2007; 13:254-261.

5. Lichtenstein GR, Olson A, Travers S, et al. Factors associated with the development of intestinal strictures or obstructions in patients with Crohn's disease. Am J Gastroenterol 2006; 101:1030-1038.

6. Oberhuber G, Stangl PC, Vogelsang H, Schober E, Herbst F, Gasche C Significant association of strictures and internal fistula formation in Crohn's disease. Virchows Arch 2000; 437:293-297.

7. Dhillon S, Loftus EV, Tremaine WJ, et al. The natural history of surgery for Crohn's disease in a population-based cohort from Olmsted County, Minnesota [abstract]. Am J Gastroenterol 2005; 100(suppl):S303.

8. Adler J, Punglia DR, Dillman JR, et al. Computed tomography enterography findings correlate with tissue inflammation, not fibrosis in resected small bowel Crohn's disease. Inflamm Bowel Dis 2012; 18:849-856.

9. Cosnes J. Can we modulate the clinical course of inflammatory bowel diseases by our current treatment strategies? Dig Dis 2009; 27:516-521.

10. Jones DW, Finlayson SR. Trends in surgery for Crohn's disease in the era of infliximab. Ann Surg 2010; 252:307-312.

11. Dillman JR, Stidham RW, Higgins PD, Moons DS, Johnson LA, Rubin JM. US elastography-derived shear wave velocity helps distinguish acutely inflamed from fibrotic bowel in a Crohn disease animal model. Radiology 2013; 267:757-766

12. Palmeri ML, Nightingale KR. Acoustic radiation force-based elasticity imaging methods. Interface Focus 2011; 1:553-564.

13. Johnson LA, Luke A, Sauder K, Moons DS, Horowitz JC, Higgins PD Intestinal fibrosis is reduced by early elimination of inflammation in a mouse model of IBD: impact of a "top-down" approach to intestinal fibrosis in mice. Inflamm Bowel Dis 2012; 18:460-471.

14. Higgins PD, Johnson LA, Sauder K, et al. Transient or persistent norovirus infection does not alter the pathology of Salmonella typhimurium induced intestinal inflammation and fibrosis in mice. Comp Immunol Microbiol Infect Dis 2011; 34:247-257.

15. Kim K, Johnson LA, Jia C, et al. Noninvasive ultrasound elasticity imaging (UEI) of Crohn's disease: animal model. Ultrasound Med Biol 2008; 34:902-912.

16. XuJ,Tripathy S, Rubin JM, et al. A new nonlinear parameter in the developed strain-to-applied strain of the soft tissues and its application in ultrasound elasticity imaging. Ultrasound Med Biol 2012; 38:511-523. 
17. Stidham RW, Xu J, Johnson LA, et al. Ultrasound elasticity imaging for detecting intestinal fibrosis and inflammation in rats and humans with Crohn's disease. Gastroenterology 2011; 141:819-826.

18. Sarvazyan AP, Urban MW, GreenleafJF.Acoustic waves in medical imaging and diagnostics. Ultrasound Med Biol 2013; 39:1133-1146.

19. Jacene HA, Ginsburg P, Kwon J, et al. Prediction of the need for surgical intervention in obstructive Crohn's disease by ${ }^{18} \mathrm{~F}$-FDG PET/CT.JNucl Med 2009; 50:1751-1759.

20. Rotemberg V, Byram B, Palmeri M, Wang M, Nightingale K. Ultrasonic characterization of the nonlinear properties of canine livers by measuring shear wave speed and axial strain with increasing portal venous pressure. J Biomech 2013; 46:1875-1881. 